\title{
A one-step reduced mechanism for near-limit hydrogen combustion with general stochiometry
}

\author{
Daniel Fernández-Galisteo \\ Department of Energy, \\ Centro de Investigaciones Energéticas, Medioambientales y Tecnológicas, Madrid, Spain \\ Adam Weiss, Antonio L. Sánchez, Forman A. Williams* \\ Department of Mechanical and Aerospace Engineering, University of California San Diego, \\ La Jolla, CA 92093-0411, USA
}

\begin{abstract}
A one-step reduced mechanism for hydrogen combustion, previously developed for fuel-lean flames, is extended to apply for all equivalence ratios under near-limit conditions by taking into consideration two additional recombination steps that are important under fuel-rich conditions. It is found that the crossover temperature that appears in the cutoff factor is smaller under fuel-rich conditions. Besides im- proving insights, the results can be beneficial in speeding computations.
\end{abstract}

Previous analyses of hydrogen-air deflagrations near the lean flammability limit $[1,2]$ have shown that corresponding burning rates are controlled by only seven elementary steps, namely the reversible shuffle reactions $\mathrm{H}+\mathrm{O}_{2} \stackrel{1}{\rightleftharpoons} \mathrm{OH}+\mathrm{O}, \mathrm{H}_{2}+\mathrm{O} \stackrel{2}{\rightleftharpoons} \mathrm{OH}+\mathrm{H}$, and $\mathrm{H}_{2}+\mathrm{OH} \stackrel{3}{\rightleftharpoons}$ $\mathrm{H}_{2} \mathrm{O}+\mathrm{H}$, the three-body reaction $\mathrm{H}+\mathrm{O}_{2}+\mathrm{M} \stackrel{4 f}{\rightarrow} \mathrm{HO}_{2}+\mathrm{M}$, and the hydroperoxyl-consumption reactions $\mathrm{HO}_{2}+\mathrm{H} \stackrel{5 f}{\rightarrow} \mathrm{OH}+\mathrm{OH}, \mathrm{HO}_{2}+\mathrm{H} \stackrel{6 f}{\rightarrow} \mathrm{H}_{2}+\mathrm{O}_{2}$, and $\mathrm{HO}_{2}+\mathrm{OH} \stackrel{7 f}{\rightarrow} \mathrm{H}_{2} \mathrm{O}+\mathrm{O}_{2}$, the last four being irreversible. Radical production by branching and radical consumption by recombination were found to be nearly in balance in the reaction layer, so that all radicals obey a steady-state approximation there, while the main species react according to the global oxidation reaction

$$
2 \mathrm{H}_{2}+\mathrm{O}_{2} \rightarrow 2 \mathrm{H}_{2} \mathrm{O}
$$

The associated $\mathrm{H}_{2}$-oxidation rate was found to be equal to that of the elementary three-body reaction $4 f$, involving the concentration of $\mathrm{H}$, which was determined by solving exactly the radical steady-state equations stemming from the 7-step mechanism, thereby providing an explicit expression for the global oxidation rate in terms of the concentration of main species

\footnotetext{
*Corresponding author

Email address: faw@ucsd.edu (Forman A. Williams)

Published Journal Article available online: https://doi.org/10.1016/j.combustflame.2019.06.018

Preprint submitted to Combustion and Flame
}

May 21, 2019 
and temperature [1]. The resulting one-step model has been successfully applied to $\mathrm{H}_{2}-\mathrm{O}_{2}$ combustion problems involving very lean mixtures, including computations of burning rates [2], flame ball structures [3], and predictions of flammability conditions [4].

This brief communication extends these previous ideas, developed for lean combustion, to systems of general stoichiometry, under conditions such that, as a result of dilution of the reactants with inert species (e.g., by exhaust-gas recirculation), the peak temperatures are not far above the crossover value at which the rate vanishes according to the reducedchemistry description, the calculated concentrations of all radicals going to zero because of the absence of low-temperature chemistry. This places the radical pool near steady state, as may be expected to occur in mild combustion [5]. The main difference from the previous development [1] lies in the needed set of elementary reactions. Away from the lean flammability limit, the three-body reactions $\mathrm{H}+\mathrm{OH}+\mathrm{M} \stackrel{8 f}{\rightarrow} \mathrm{H}_{2} \mathrm{O}+\mathrm{M}$ and $\mathrm{H}+\mathrm{H}+\mathrm{M} \stackrel{9 f}{\rightarrow}$ $\mathrm{H}_{2}+\mathrm{M}$, which were discarded in the previous analyses of lean combustion $[1,2]$, become important and must be retained for computations of burning rates [6]. Consequently, if general stoichiometry is to be addressed, then a total of nine elementary reactions must be considered as the starting point in the chemical-kinetic reduction process.

The derivation of the reduced chemistry parallels that presented earlier [1], so that details may be omitted for brevity. Linear combinations of the production rates $\dot{C}_{i}$ (mols per unit volume per unit time) of the seven different reactive species $i=\left(\mathrm{H}_{2}, \mathrm{O}_{2}, \mathrm{H}_{2} \mathrm{O}, \mathrm{H}, \mathrm{O}, \mathrm{OH}, \mathrm{HO}_{2}\right)$ involved in the nine elementary reactions lead to the expressions $-\dot{C}_{\mathrm{H}_{2}} / 2=-\dot{C}_{\mathrm{O}_{2}}=$ $\dot{C}_{\mathrm{H}_{2} \mathrm{O}} / 2=\omega_{4 f}+\omega_{8 f}+\omega_{9 f}$ when the anticipated small values of the radical concentrations are neglected. These equations indicate that oxidation of the fuel occurs according to the overall reaction (1), with the associated rate

$$
\omega=\omega_{4 f}+\omega_{8 f}+\omega_{9 f}=k_{4 f} C_{\mathrm{M}_{4}} C_{\mathrm{O}_{2}} C_{\mathrm{H}}+k_{8 f} C_{\mathrm{M}_{8}} C_{\mathrm{OH}} C_{\mathrm{H}}+k_{9 f} C_{\mathrm{M}_{9}} C_{\mathrm{H}}^{2},
$$

where $k_{j}$ denotes the reaction-rate constant of reaction $j$, and $C_{i}$ and $C_{\mathrm{M}_{j}}$ denote the concentration of species $i$ and the effective third-body concentration, the latter evaluated for each reaction $j=(4 f, 8 f, 9 f)$ with account taken of its specific chaperon efficiencies. The present extension serves to add the last two terms in this expression.

The concentrations of $\mathrm{OH}$ and $\mathrm{H}$ in (2) can be obtained in terms of the concentrations of $\mathrm{O}_{2}, \mathrm{H}_{2}, \mathrm{H}_{2} \mathrm{O}$ and the temperature $T$ by solving the nonlinear set of equations found by equating to zero the production rates of $\mathrm{O}, \mathrm{OH}, \mathrm{H}$, and $\mathrm{HO}_{2}$. The algebraic manipulation is facilitated by noting that in the reaction layer the conditions $k_{8 f} C_{\mathrm{M}_{8}} C_{\mathrm{H}} \ll k_{3 f} C_{\mathrm{H}_{2}}$ and $k_{9 f} C_{\mathrm{M}_{9}} C_{\mathrm{H}} \ll k_{3 b} C_{\mathrm{H}_{2} \mathrm{O}}$ are always satisfied, so that the effects of reactions $8 f$ and $9 f$ can be neglected when computing the relative $\mathrm{OH}-\mathrm{to}-\mathrm{H}$ ratio. Adoption of this approximation leads to the explicit expressions

$$
C_{\mathrm{OH}}=\frac{1}{H} \frac{k_{2 f} C_{\mathrm{H}_{2}}}{k_{1 b}}\left(\frac{k_{1 f}}{\alpha k_{4 f} C_{\mathrm{M}_{4}}}-1\right)
$$

and

$$
C_{\mathrm{H}}=\frac{1}{G H} \frac{k_{2 f} k_{3 f} C_{\mathrm{H}_{2}}^{2}}{k_{1 b} k_{4 f} C_{\mathrm{M}_{4}} C_{\mathrm{O}_{2}}}\left(\frac{k_{1 f}}{\alpha k_{4 f} C_{\mathrm{M}_{4}}}-1\right),
$$


where

$$
\begin{aligned}
G & =\frac{1+\gamma_{3 b}}{2}+\frac{f}{2}\left\{\left[1+2\left(3+\gamma_{3 b}\right) / f+\left(1+\gamma_{3 b}\right)^{2} / f^{2}\right]^{1 / 2}-1\right\}, \\
\alpha & =\frac{k_{6 f} f /\left(k_{5 f}+k_{6 f}\right)+G}{f+G} \\
H & =\frac{1}{2}(1+A B)\left\{1+\left[1+\frac{4(1+A) B}{(1+A B)^{2}}\left(\frac{k_{1 f}}{\alpha k_{4 f} C_{\mathrm{M}_{4}}}-1\right)\right]^{1 / 2}\right\}
\end{aligned}
$$

with

$$
\begin{aligned}
& f=\frac{k_{5 f}+k_{6 f}}{k_{7 f}} \frac{k_{3 f}}{k_{4 f} C_{\mathrm{M}_{4}}} \frac{C_{\mathrm{H}_{2}}}{C_{\mathrm{O}_{2}}}, \quad \gamma_{3 b}=\frac{k_{3 b} C_{\mathrm{H}_{2} \mathrm{O}}}{k_{4 f} C_{\mathrm{M}_{4}} C_{\mathrm{O}_{2}}} \\
& A=\frac{k_{8 f} C_{\mathrm{M}_{8}}}{k_{2 b}}+\frac{k_{9 f} C_{\mathrm{M}_{9}}}{k_{2 b}} \frac{k_{7 f}}{k_{5 f}+k_{6 f}} \frac{f}{G}, \quad B=\frac{k_{7 f}}{k_{5 f}+k_{6 f}} \frac{k_{2 b} k_{2 f}}{k_{1 b} k_{3 f}} \frac{f}{\alpha} .
\end{aligned}
$$

The systematically derived expressions (3) and (4) are seen to include a cutoff factor (the term in parenthesis) associated with the existence of a chemical-kinetic crossover temperature $T_{c}$ [6], defined by the condition $k_{1 f}=\alpha k_{4 f} C_{\mathrm{M}_{4}}$. where $\alpha$ is to be evaluated from (6) with use made of (5) and (8)], below which low-temperature chemistry prevails and the formulas fail. The parameter $A$ carries the effect of the radical-radical recombination reactions $8 f$ and $9 f$; correspondingly, for $A=0$ the steady-state expressions (3) and (4) reduce exactly to those developed earlier [1].

The one-step mechanism was tested in numerical computations employing the reactionrate parameters of the San Diego mechanism [7], with results given in Fig. 1 for steady planar deflagrations and in Fig. 2 for steady continuously stirred flow reactors. Figure 1 shows the variation with equivalence ratio $\phi$ of the flame propagation velocity $v_{l}$ computed with detailed chemistry [6] and with the explicit one-step mechanism for $\mathrm{H}_{2}-\mathrm{O}_{2}-\mathrm{N}_{2}$ mixtures with two different degrees of dilution. The figure also shows the corresponding variation of the one-step adiabatic temperature $T_{a}$ and crossover temperature $T_{c}$, the latter obtained for each value of $\phi$ by solving $k_{1 f}=\alpha k_{4 f} C_{\mathrm{M}_{4}}$ employing the corresponding equilibrium composition. Flame propagation requires that $T_{a}>T_{c}$, as needed for existence of radicals in the reaction layer, so that the condition $T_{a}=T_{c}$ determines the critical values of $\phi$ associated with the lean and rich flammability limits, at which the flame propagation velocity is calculated to vanish, as confirmed by the results in Fig. 1.

It can be shown from the formulas that the reaction rate simplifies to

$$
\omega=\left(\frac{k_{1 f}}{k_{4 f} C_{\mathrm{M}_{4}}}-1\right) \frac{k_{2 f} k_{3 f} / k_{1 b}}{1+\frac{k_{3 b} C_{\mathrm{H}_{2} \mathrm{O}}}{k_{4 f} C_{\mathrm{M}_{4}} C_{\mathrm{O}_{2}}}} C_{\mathrm{H}_{2}}^{2}
$$

near the lean limit and to

$$
\omega=\left(\frac{k_{1 f}}{k_{4 f} C_{\mathrm{M}_{4}}}-1+\frac{k_{5 f} /\left(k_{5 f}+k_{6 f}\right)}{1+\frac{k_{7 f}}{k_{5 f}+k_{6 f}} \frac{k_{3 b} C_{\mathrm{H}_{2}}}{k_{3 f} C_{\mathrm{H}_{2} \mathrm{O}}}}\right) \frac{\left(k_{4 f} C_{\mathrm{M}_{4}}\right)^{2}}{\frac{k_{3 b} C_{\mathrm{H}_{2} \mathrm{O}}}{k_{3 f} C_{\mathrm{H}_{2}}} k_{8 f} C_{\mathrm{M}_{8}}+k_{9 f} C_{\mathrm{M}_{9}}} C_{\mathrm{O}_{2}}^{2}
$$


near the rich limit, revealing that the overall rate is second order in the deficient reactant in the vicinity of both limits. In Fig. 1 the value of $T_{c}$ is seen to be lower on the rich side than on the lean side, in agreement with the fact that the value of $\alpha=1-k_{5 f} /\left\{k_{5 f}+k_{6 f}+\right.$ $\left.k_{7 f}\left[\left(k_{3 b} C_{\mathrm{H}_{2}}\right) /\left(k_{3 f} C_{\mathrm{H}_{2} \mathrm{O}}\right)\right]\right\}$ in the cutoff factor of the rich-limit expression (11) is smaller than the value $\alpha=1$ found in the lean-limit expression (10). The change of $T_{c}$ at stoichiometric conditions becomes increasingly abrupt with increasing dilution because of the associated decrease in radical concentrations, leading, for example, to a nearly discontinuous change of $f$ in (8) from near zero to near infinity as $\phi$ increases through unity. This results, for example, in sharp kinks at stoichiometry in curves (not shown) of $v_{l}$ as functions of $\phi$ for fixed flame temperatures (the rich-side burning velocities rising rapidly because of the smaller $T_{c}$ ).

The one-step description is strictly valid for flames with peak temperatures lying close to the crossover value, as is needed for accuracy of the steady-state approximations. With sufficient dilution, this condition is achieved for all flammable mixtures, as can be seen in the results presented in Fig. 1a for a highly diluted mixture with $Y_{\mathrm{O}_{2} u} /\left(Y_{\mathrm{N}_{2} u}+Y_{\mathrm{O}_{2} u}\right)=$ 0.087, where $Y_{\mathrm{O}_{2} u}$ and $Y_{\mathrm{N}_{2} u}$ represent the oxygen and nitrogen mass fractions in the unburnt mixture. At this dilution the predicted burning rate of the one-step mechanism differs by less than $5 \%$ from that of the detailed-chemistry computations, and the differences in computational predictions are seen to be comparable with the differences between sets of experimental measurements for these mixtures. For $\mathrm{H}_{2}$-air flames $\left[Y_{\mathrm{O}_{2} u} /\left(Y_{\mathrm{N}_{2} u}+Y_{\mathrm{O}_{2} u}\right) \simeq\right.$ 0.232], shown in Fig. 1b, near-crossover equilibrium temperatures arise only near the lean and rich flammability limits, although the range of composition over which the one-step mechanism provides reasonable accuracy is seen to be fairly broad, especially for fuel-lean mixtures, with overpredictions of $v_{l}$ remaining smaller than approximately $25 \%$ for $\phi \lesssim 0.9$. The predictive capability of the new model in connection with $\mathrm{H}_{2}$-air combustion is therefore significantly better than that of the previous one-step description, the improvement being attributable to inclusion of the additional recombination reactions $8 \mathrm{f}$ and $9 \mathrm{f}$.

The stirred-reactor results shown in Fig. 2 were obtained from the nonlinear set of algebraic equations that determine the variation of species mass fractions $Y_{i}$ and temperature $T$ from their unburnt values $Y_{i_{u}}$ and $T_{u}$, as given by

$$
\rho\left(Y_{i_{u}}-Y_{i}\right)+t_{\mathrm{R}} M_{i} \dot{C}_{i}=0 \quad \text { and } \quad \rho c_{p}\left(T_{u}-T\right)+t_{\mathrm{R}} \sum h_{i}^{o} M_{i} \dot{C}_{i}=0,
$$

where $c_{p}$ is the specific heat at constant pressure (assumed to be constant), $\rho$ is the density, and $M_{i}$ and $h_{i}^{o}$ are the molecular mass and the enthalpy of formation of species $i$. These equations were solved with $T_{u}=298 \mathrm{~K}$ for stoichiometric $\mathrm{H}_{2}-\mathrm{O}_{2}-\mathrm{N}_{2}$ mixtures with three different levels of nitrogen dilution, selected to give adiabatic temperatures $T_{a}=(1000,1100,1200) \mathrm{K}$. The resulting curves include a near-equilibrium branch approaching $T=T_{a}$ and $Y_{\mathrm{O}_{2}}=Y_{\mathrm{H}_{2}}=Y_{\mathrm{H}}=0$ as $t_{\mathrm{R}} \rightarrow \infty$ and an unstable branch approaching $T=T_{c}$ and $Y_{\mathrm{H}}=0$, having nonzero values of $Y_{\mathrm{O}_{2}}$ and $Y_{\mathrm{H}_{2}}$ as $t_{\mathrm{R}} \rightarrow \infty$, with a turning point at a minimum value of $t_{\mathrm{R}}$, characterizing extinction. The one-step approximation is seen to describe both branches with reasonable accuracy, as well as the extinction residence times, the departures becoming larger as the steady-state approximation degrades at higher values of $T_{a}$ and as $t_{\mathrm{R}}$ decreases, allowing less time for achievement of the steady state. Detailed 
chemistry could extend these C-shaped curves to S shapes, introducing an ignition turning point at exceedingly large values of $t_{\mathrm{R}}$.

This new one-step approximation does not properly describe autoignition processes because it neglects the time required for achieving the steady states, but it can facilitate descriptions of other aspects of mild combustion. Moreover, relevant simplified analyses of cellular-flame phenomena under fuel-lean conditions and of pulsating-flame behaviors under fuel-rich conditions may well be based on this one-step approximation, thereby complementing existing computational investigations $[8,9]$. Future utility of this new result may thus be extensive.

\section{Acknowledgements}

This work was supported by the US AFOSR Grant No. FA9550-16-1-0443. DFG was supported by project \#ENE2015-65852-C2-2-R (MINECO/FEDER, EU).

[1] D. Fernández-Galisteo, A. Sánchez, A. Liñán, F. Williams, One-step reduced kinetics for lean hydrogenair deflagration, Combust. Flame 156 (5) (2009) 985-996.

[2] D. Fernández-Galisteo, A. Sánchez, A. Liñán, F. Williams, The hydrogen-air burning rate near the lean flammability limit, Combust. Theor. Model. 13 (4) (2009) 741-761.

[3] E. Fernández-Tarrazo, A. L. Sánchez, A. Liñán, F. Williams, The structure of lean hydrogen-air flame balls, Proc. Combust. Inst. 33 (1) (2011) 1203-1210.

[4] E. Fernández-Tarrazo, A. L. Sánchez, A. Liñán, F. A. Williams, Flammability conditions for ultra-lean hydrogen premixed combustion based on flame-ball analyses, Int. J. Hydrogen Energ. 37 (2) (2012) 1813-1825.

[5] A. Cavaliere, M. de Joannon, Mild combustion, Prog. Energy Combust. Sci. 30 (4) (2004) 329-366.

[6] A. L. Sánchez, F. A. Williams, Recent advances in understanding of flammability characteristics of hydrogen, Prog. Energy Combust. Sci. 41 (2014) 1-55.

[7] The San Diego Mechanism: Chemical-Kinetic Mechanisms for Combustion Applications, https:// web.eng.ucsd.edu/mae/groups/combustion/mechanism.html, version: 2016-12-14.

[8] J. F. Grcar, A new type of steady and stable, laminar, premixed flame in ultra-lean, hydrogen-air combustion, Proc. Combust. Inst. 32 (1) (2009) 1011-1018.

[9] A. Korsakova, V. Gubernov, A. Kolobov, V. Bykov, U. Maas, Stability of rich laminar hydrogen-air flames in a model with detailed transport and kinetic mechanisms, Combust. Flame 163 (2016) 478-486.

[10] R. J. Kee, J. F. Grcar, M. D. Smooke, J. A. Miller, E. Meeks, PREMIX: a Fortran program for modeling steady laminar one-dimensional premixed flames, Sandia National Laboratories Report SAND85-8249.

[11] F. Egolfopoulos, C. K. Law, An experimental and computational study of the burning rates of ultralean to moderately-rich $\mathrm{H}_{2} / \mathrm{O}_{2} / \mathrm{N}_{2}$ laminar flames with pressure variations, Proc. Combust. Inst. 23 (1) (1991) 333-340.

[12] R. Hermanns, A. A. Konnov, R. Bastiaans, L. De Goey, Laminar burning velocities of diluted hydrogenoxygen-nitrogen mixtures, Energy Fuels 21 (4) (2007) 1977-1981.

[13] V. R. Kishore, R. Muchahary, A. Ray, M. Ravi, Adiabatic burning velocity of $\mathrm{H}_{2}-\mathrm{O}_{2}$ mixtures diluted with $\mathrm{CO}_{2} / \mathrm{N}_{2}$ /Ar, Int. J. Hydrogen Ener. 34 (19) (2009) 8378-8388. 

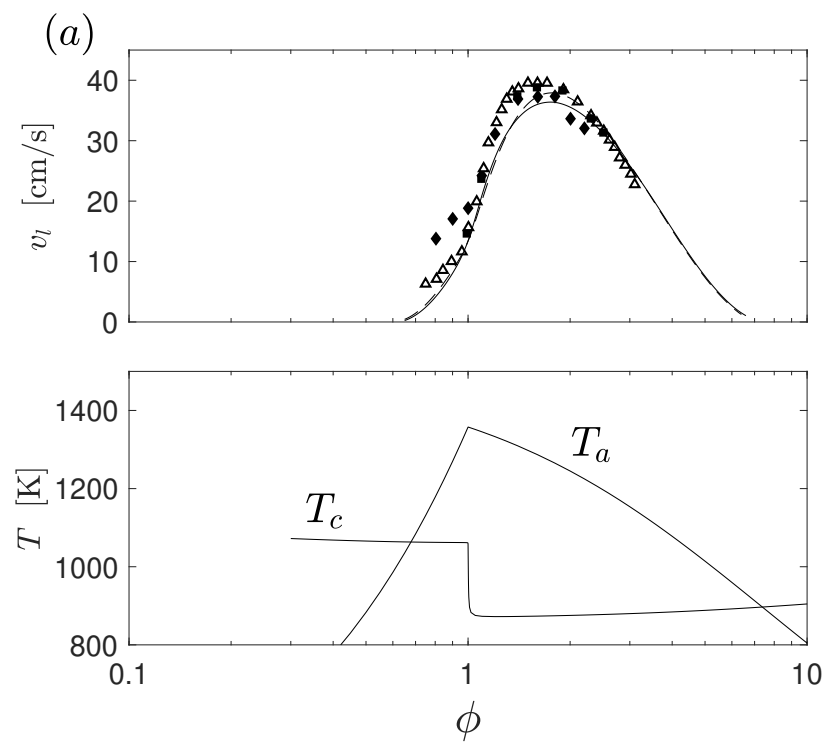

(b)
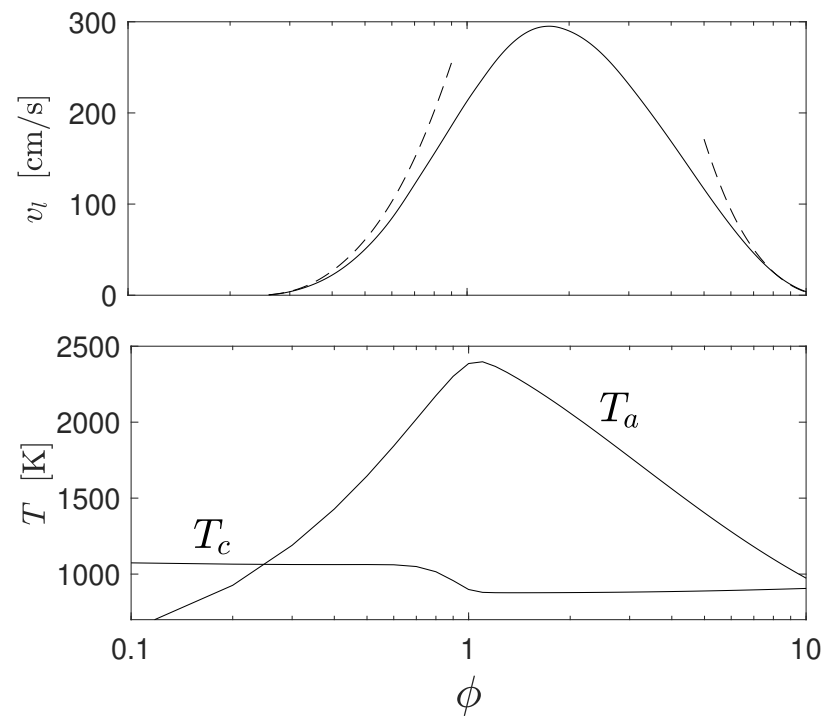

Figure 1: The variation with equivalence ratio $\phi$ of the laminar propagation velocity $v_{l}$ of $\mathrm{H}_{2}-\mathrm{O}_{2}-\mathrm{N}_{2}$ flames, as obtained from integrations of the premixed-flame equations [10] with mixture-average transport, for the 20-step San Diego mechanism (solid curves) and for the one-step reaction (dashed curves), at 1 atmosphere, for an unburnt temperature of $T_{u}=300 \mathrm{~K}$. Also shown are the adiabatic temperature $T_{a}$, obtained from equilibrium computations, and the crossover temperature $T_{c}$, obtained from $k_{1 f}=\alpha k_{4 f} C_{\mathrm{M}_{4}}$ with $\alpha$ and $C_{\mathrm{M}_{4}}$ evaluated for the equilibrium composition. Subfigure (a), pertaining to high dilution, also includes experimental measurements (diamonds: [11], triangles: [12], squares: [13]), while (b) corresponds to hydrogen-air mixtures. 

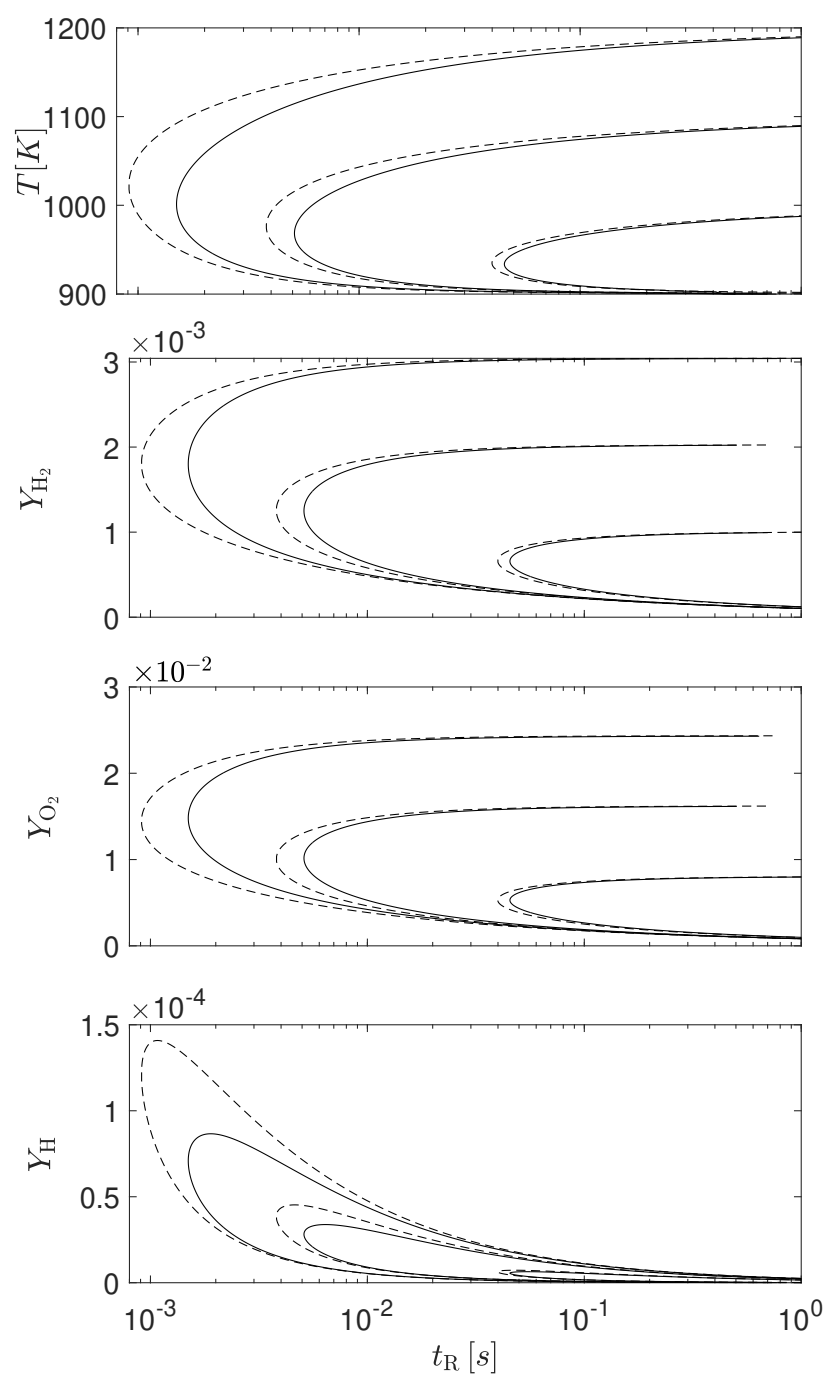

Figure 2: The variation with residence time of the temperature and of the mass fractions of reactants and $\mathrm{H}$ atoms in a continuous well-stirred reactor as obtained with 9 elementary steps (solid curves) and with the one-step description (dashed curves) for a stoichiometric $\mathrm{H}_{2}-\mathrm{O}_{2}-\mathrm{N}_{2}$ mixture with an inlet (subscript $u$ ) temperature of $298 \mathrm{~K}$ at 1 atmosphere. The three pairs of curves in each plot correspond to mixtures with $Y_{\mathrm{O}_{2} u} /\left(Y_{\mathrm{N}_{2} u}+Y_{\mathrm{O}_{2} u}\right)=(0.0575,0.0658,0.0740)$ yielding corresponding adiabatic flame temperatures $T_{a}=(1000,1100,1200) \mathrm{K}$. 